\title{
Searches for leptoquarks and excited leptons with the ATLAS detector
}

\author{
Simon Viel ${ }^{* \dagger}$ \\ on behalf of the ATLAS Collaboration \\ Lawrence Berkeley National Lab. (US) \\ E-mail: svielecern.ch
}

Searches for pair-produced first-generation and second-generation leptoquarks and for excited muons are presented, in final states consisting of two charged leptons and at least two jets. The studies reported use data recorded by the ATLAS experiment at the Large Hadron Collider in 2015 at $\sqrt{s}=13 \mathrm{TeV}$, and in 2012 at $\sqrt{s}=8 \mathrm{TeV}$.

38th International Conference on High Energy Physics

3-10 August 2016

Chicago, USA

\footnotetext{
* Speaker.

${ }^{\dagger}$ The author acknowledges support from the U.S. Department of Energy and the Natural Sciences and Engineering Research Council of Canada.
} 


\section{Introduction}

The observed similarity of the quark and lepton sectors in the Standard Model of particle physics seems to point at a connection between the two. Many hypotheses of physics beyond the Standard Model, such as grand unified theories and models with quark and lepton substructure, are possible ways to make this connection. For example, models involving a grand unified symmetry group where the primordial symmetry is broken down to the symmetry group of the Standard Model via $S U(4)$ [1] or $S U(5)$ [2] predict leptoquarks, which carry both quark and lepton quantum numbers. In general, leptoquarks predicted by these theories are ruled out at the $\mathrm{TeV}$ scale, as they would mediate processes such as proton decay, $K \rightarrow e \mu$, etc. However, the experimental bounds on leptoquark models due to the non-observation of these processes can be evaded with a number of theoretical constraints. An example of such a model is the minimal Buchmüller-Rückl-Wyler (mBRW) model [3]. Lepton and quark substructure could manifest itself via the observation of excited fermion states [4]. If their mass is near the TeV scale, excited fermions and leptoquarks could be produced at the Large Hadron Collider.

The ATLAS collaboration [5] has performed searches for first-generation and secondgeneration leptoquarks [6] in $3.2 \mathrm{fb}^{-1}$ of $p p$ collision data recorded at a centre-of-mass energy $\sqrt{s}=13 \mathrm{TeV}$ in 2015, and for excited muons [7] in the full $p p$ collision dataset recorded in 2012 $\left(20.3 \mathrm{fb}^{-1}\right.$ at $\left.\sqrt{s}=8 \mathrm{TeV}\right)$.

All the subsystems of the ATLAS detector are used in these searches. The inner detector, consisting of silicon pixels, silicon strips and straw tube gas detectors, is used to reconstruct charged particle tracks and vertices. A solenoid magnet provides a homogeneous field of $2 \mathrm{~T}$, allowing to measure the momentum of these charged particles using track curvature. Electromagnetic and hadronic calorimeters are responsible for reconstructing the particle showers that occur when electrons, photons and hadrons interact with absorbing material in these detectors. The electromagnetic calorimeter is made of liquid argon and lead, while the central part of the hadronic calorimeter is composed of scintillating tiles and steel, and the forward calorimeters are made of liquid argon, copper and tungsten. Outside the calorimeters, toroid magnets provide the field for the muon spectrometer, which consists of three sets of drift tubes and cathode strip chambers allowing for the precise reconstruction of muon tracks, as well as resistive-plate and thin-gap chambers providing triggering capabilities.

\section{Analysis strategy}

The first analysis looks for pair-produced leptoquarks each decaying into a lepton and a quark, while the second explores the possibility of an excited composite muon produced and decaying via $\mu \mu q \bar{q}$ contact interactions $\left(q \bar{q} \rightarrow \mu \mu^{*} \rightarrow \mu \mu q \bar{q}\right)$. In both cases, the final state therefore consists of two high-momentum electrons or muons, in addition to at least two hadronic jets.

Electrons are measured as clusters of electromagnetic-calorimeter cells with significant energy deposits matched to tracks reconstructed in the inner detector. The energy of high-energy electrons is well-measured by the electromagnetic calorimeter, as the dominant uncertainties in this case come from small instrumental effects that do not scale with energy, and the electron direction is given by the associated track. The main challenge in the electron channel is particle identification. 
In order to select electron candidates against a large background of hadronic jets and converted photons, electron candidates are required to satisfy requirements on the cluster shape, leakage into the hadronic calorimeter, quality of the inner detector track and matching to the cluster, as well as the isolation of the electron candidate from other activity in the event.

In the muon channel, momentum is measured independently in the inner detector and muon spectrometer using the curvature of muon tracks. At very high momentum, this curvature is very small and the momentum measurement becomes dominated by the detector alignment and intrinsic resolution. It is therefore imperative to use only the best-aligned muon detector chambers for these searches, with stringent track quality requirements. The measurements from the inner detector and muon spectrometer are combined in a fit, taking into account multiple-scattering effects in the detector material as well as energy loss in the calorimeter. Muons originating from hadron decays are rejected using a track-based isolation requirement.

Jets are reconstructed from clusters of energy deposits in the calorimeter using the anti- $k_{t}$ algorithm [8] with radius parameter $R=0.4$. Calibrations derived from simulated data and in situ measurements are applied as a function of jet energy and direction. Requirements on the jet energy and the quality of the measurement are applied, and an overlap ambiguity resolution algorithm is applied to all measured objects in the event.

\section{Search for scalar leptoquarks at $\sqrt{s}=13 \mathrm{TeV}$}

The benchmark signal model considered in the search for leptoquarks is the mBRW model [3], which predicts three generations of scalar leptoquarks carrying fermion number 0 or 2 . Lepton number and baryon number separately conserved, ensuring the stability of the proton. The leptoquark couplings to fermions are purely chiral and flavour-diagonal: therefore the main signatures to which this search is sensitive are the pair-production of first-generation leptoquarks decaying to $e d$ or $e u$, and of second-generation leptoquarks decaying to $\mu s$ or $\mu c$. Nevertheless this search is designed to be as model-independent as possible, by imposing no requirement on lepton charge and hadronic jet flavour, retaining some sensitivity to leptoquark couplings across fermion generations.

Based on the characteristics of the leptoquark signal, the main discriminating variables in this search are the following:

- High lepton invariant mass: $m_{\ell \ell}>130 \mathrm{GeV}$

- High scalar transverse momentum sum of the two leptons and two leading jets: $S_{\mathrm{T}}>600 \mathrm{GeV}$

- High minimum invariant mass of lepton-jet pairs $m_{\mathrm{LQ}}^{\min }$, where the lepton-jet pairs are chosen such that the invariant mass difference $\left|m_{\mathrm{LQ}}^{\max }-m_{\mathrm{LQ}}^{\min }\right|$ is minimized.

The main backgrounds in this search are $t \bar{t}$ and Drell-Yan $\left(Z / \gamma^{*}\right)$ production in final states with two charged leptons, followed by diboson and single-top processes. These backgrounds are evaluated using Monte Carlo simulation, with the production cross-sections taken from theoretical predictions at next-to-leading order and next-to-next-to-leading order. In the electron channel, there is also a small contribution from jets and mis-identified converted photons: data-driven strategies are employed to estimate the rate of these backgrounds after the event selection. 
The normalizations of the main two background sources are constrained in dedicated control regions, defined by reversing one of the criteria defining the signal region. Events in the $t \bar{t}$ control region are selected by requiring exactly one electron and one muon, and the $Z / \gamma^{*}$ control region is defined by requiring $m_{\ell \ell}$ to be on the $Z$ peak: $70 \mathrm{GeV}<m_{\ell \ell}<110 \mathrm{GeV}$. The background shape observed in control region data is well-described by simulated events, and the normalization factors resulting from a combined fit of control regions and signal regions are consistent with unity. Validation regions are defined using the full event selection but inverting the requirement on $S_{\mathrm{T}}$, and there is good agreement between the data and background predictions in both channels.

Figure 1 shows predicted and observed event yields in the signal regions of this search as a function of $m_{\mathrm{LQ}}^{\min }$. The main uncertainties in this search come from theoretical background predictions in the leptoquark phase-space. Experimental uncertainties, mainly from jet calibration, lepton identification and the luminosity measurement, are also considered. The statistical analysis is performed using a profile-likelihood fit of background plus signal templates as a function of $m_{\mathrm{LQ}}^{\mathrm{min}}$ to the data, and results are consistent with expectations from the Standard Model.

Limits are set on the leptoquark pair-production cross-section $\left(\sigma_{\mathrm{LQ}}\right)$ times branching ratio to quark and charged lepton squared $\left(\beta^{2}\right)$ at $95 \%$ confidence level (CL). These limits are shown in Figure 2 for both leptoquark generations separately as a function of the leptoquark mass $m_{\mathrm{LQ}}$. Mass limits based on cross-sections from the benchmark mBRW model with $\beta=1$ are $m_{\mathrm{LQ}}>1100 \mathrm{GeV}$ and $m_{\mathrm{LQ}}>1050 \mathrm{GeV}$.
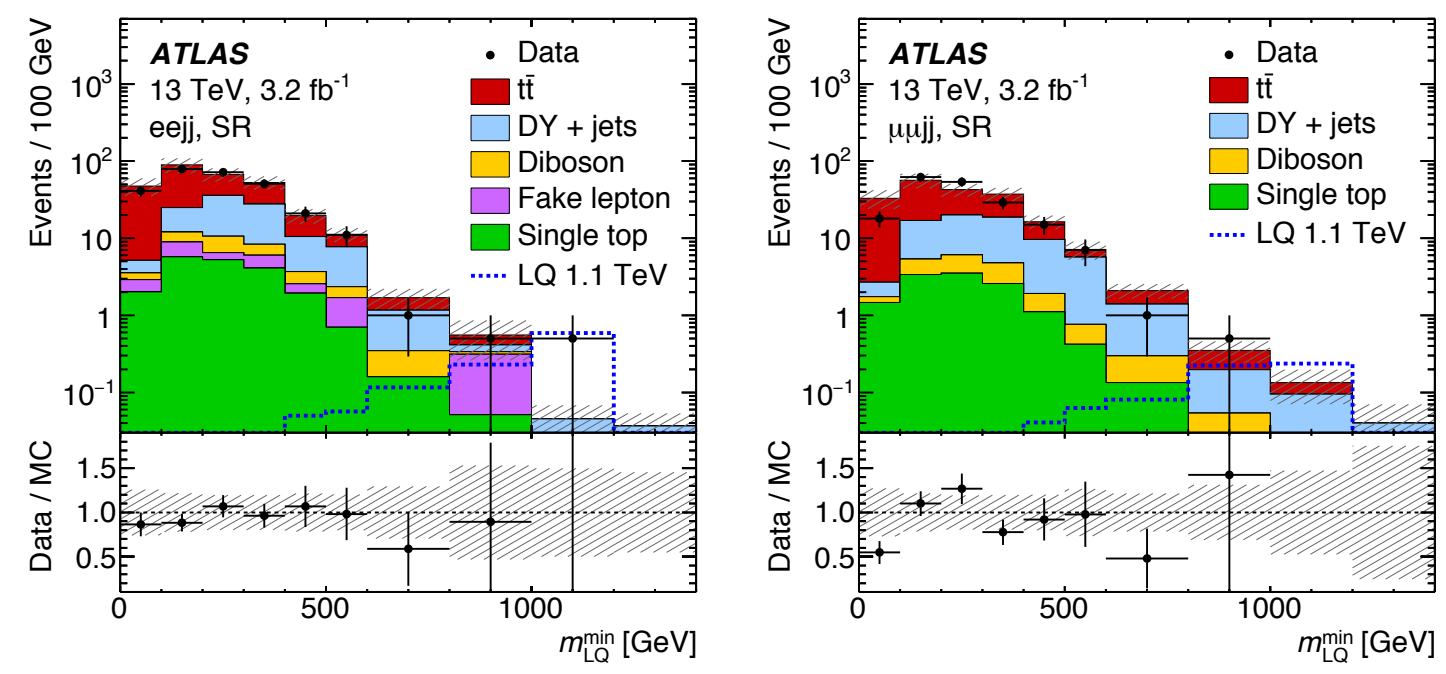

Figure 1: Distribution of the minimum leptoquark invariant mass $m_{\mathrm{LQ}}^{\min }$ in the signal region of the search for first-generation (left) and second-generation (right) leptoquarks. The two dominant backgrounds, $Z / \gamma^{*}$ and $t \bar{t}$, are shown after normalization from the fit. An example signal with $m_{\mathrm{LQ}}=1.1 \mathrm{TeV}$ is also shown. The bottom inset shows the ratio of observed to expected event yields, with the grey band representing the total systematic uncertainty on the latter. [6] 

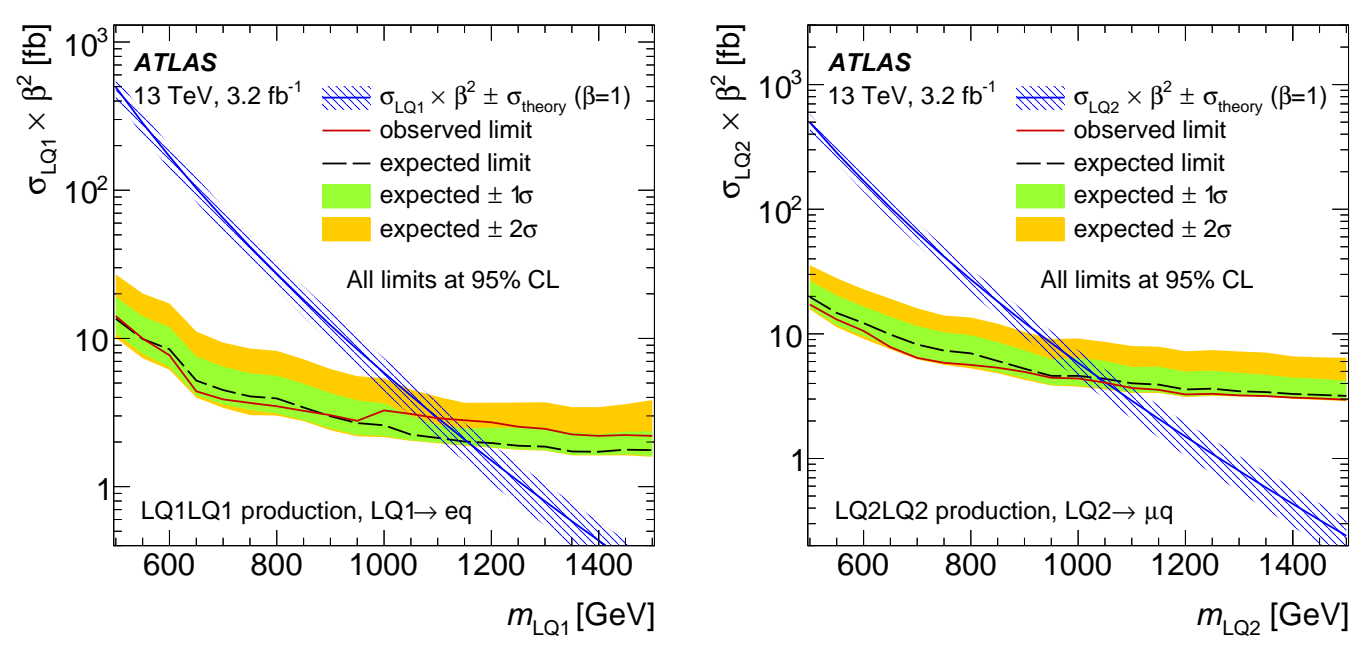

Figure 2: Limits at 95\% CL on the leptoquark signal cross-section times the square of the branching ratio as a function of $m_{\mathrm{LQ}}$ for first-generation (left) and second-generation (right) leptoquarks. [6]

\section{Search for excited muons at $\sqrt{s}=8 \mathrm{TeV}$}

The search for an excited composite muon $\mu^{*}$ via $\mu \mu q \bar{q}$ contact interactions is motivated by the fact that the decay mode $\mu^{*} \rightarrow \mu q \bar{q}$ is the dominant one when the mass of the lightest excited muon state $m_{\mu^{*}}$ is comparable to the compositeness scale $\Lambda$ [4], as illustrated in Figure 3 . A second, opposite-sign final-state muon comes from the production vertex $q \bar{q} \rightarrow \mu \mu^{*}$. At high values of $m_{\mu^{*}} / \Lambda$, the search in this $\mu \mu j j$ final state is more sensitive than the traditional search $\mu^{*} \rightarrow \mu \gamma$.

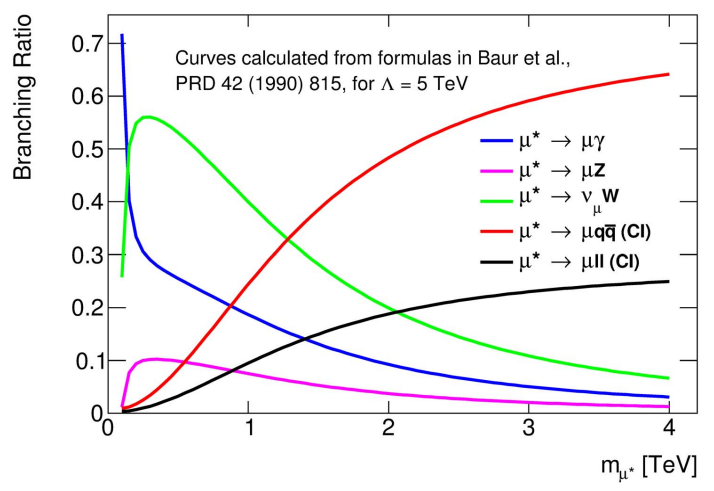

Figure 3: Expected $\mu^{*}$ branching ratios as a function of $m_{\mu^{*}}$, for $\Lambda=5 \mathrm{TeV}$. Except for threshold effects, this graph scales with $m_{\mu^{*}} / \Lambda$. [4, 7]

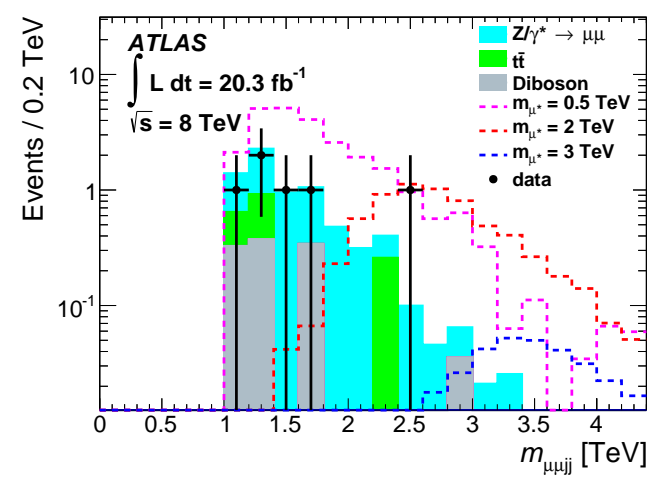

Figure 4: Distribution of $m_{\mu \mu j j}$ for signal region \#2. Three representative signal distributions are shown for $\Lambda=5 \mathrm{TeV}$. [7]

The analysis strategy is very similar to the leptoquark search in the same final state, and the main discriminating variables are $S_{\mathrm{T}}$, the dimuon invariant mass $m_{\mu \mu}$, and the four-object invariant mass $m_{\mu \mu j j}$. Three-object invariant mass combinations are found to bring no further improvement to the search sensitivity. The main backgrounds are estimated using simulated events, and the $Z / \gamma^{*}$ and $t \bar{t}$ normalizations are constrained in control regions defined as described above. 
The search sensitivity is optimized for different $m_{\mu^{*}}$ considered, by defining 10 non-orthogonal signal regions. As $m_{\mu^{*}}$ increases, requirements on $S_{\mathrm{T}}$ and $m_{\mu \mu j j}$ are tightened, and the requirement on $m_{\mu \mu}$ is allowed to become looser while remaining above the $Z / \gamma^{*}$ control region boundary at $110 \mathrm{GeV}$. An example is signal region \#2, shown in Figure 4, optimized for $m_{\mu^{*}}=300-500 \mathrm{GeV}$ : the corresponding requirements are $m_{\mu \mu}>550 \mathrm{GeV}, S_{\mathrm{T}}>900 \mathrm{GeV}$ and $m_{\mu \mu j j}>1 \mathrm{TeV}$.

No significant excess above Standard Model predictions is observed in any of the signal regions. Figure 5 displays limits set on the $\mu^{*}$ cross-section times branching ratio and on $\Lambda$ as a function of $m_{\mu^{*}}$. Observed limits on $\Lambda$ in the $\mu \mu j j$ channel are the best for $1.1 \mathrm{TeV}<m_{\mu^{*}}<2.1 \mathrm{TeV}$.
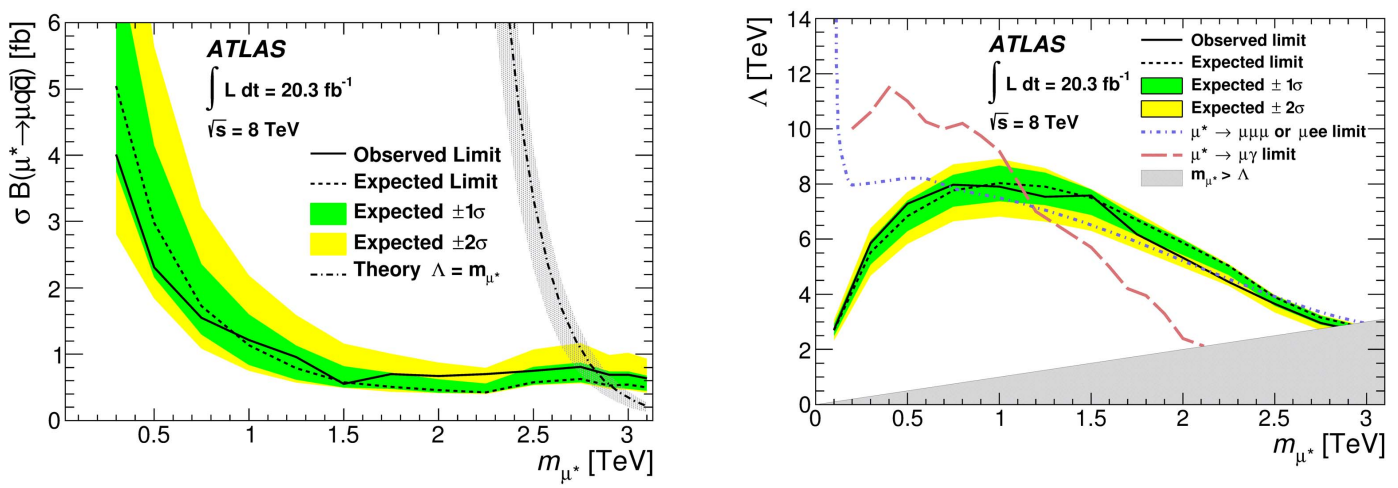

Figure 5: Limits at 95\% CL as a function of $m_{\mu^{*}}$ : upper limits on the excited muon cross-section times branching ratio (left) and lower limits on the compositeness scale $\Lambda$ (right). [7]

\section{Conclusion}

Searches for leptoquarks and excited muons in final states with two leptons and two jets are performed with the ATLAS experiment. No significant excess is found above Standard Model predictions, and limits are set on benchmark models. The analysis of more than $30 \mathrm{fb}^{-1}$ of data collected at $\sqrt{s}=13 \mathrm{TeV}$ in 2016 is currently ongoing, entering unexplored territory in the search for these new possible states of matter.

\section{References}

[1] J. C. Pati and A. Salam, Phys. Rev. D 10 (1974) 275, Erratum: Phys. Rev. D 11 (1975) 703

[2] H. Georgi and S. L. Glashow, Phys. Rev. Lett. 32 (1974) 438

[3] W. Buchmüller, R. Rückl and D. Wyler, Phys. Lett. B 191 (1987) 442, Erratum: Phys. Lett. B 448 (1999) 320

[4] U. Baur, M. Spira and P. M. Zerwas, Phys. Rev. D 42 (1990) 815

[5] ATLAS Collaboration, JINST 3 (2008) S08003

[6] ATLAS Collaboration, New J. Phys. 18 (2016) 093016

[7] ATLAS Collaboration, New J. Phys. 18 (2016) 073021

[8] M. Cacciari, G. P. Salam and G. Soyez, J. High Energy Phys. 04 (2008) 063 